\title{
A Lewis Acid-Lewis Base Bifunctional Catalyst from a New Mixed Ligand
}

Yun-Ming Lin*, Julie Boucau, Zhongtao Li, Virginie Casarotto, Jinzhen Lin, Annie, N. Nguyen, Jessica Ehrmantraut Department of Chemistry, University of Toledo

2801 Bancroft St., Toledo, OH, 43606

\section{Supporting Information}

General: All reactions were carried out under Ar using standard Schlenk techniques unless otherwise noted. Methylene chloride and diisopropylethylamine were distilled from calcium hydride. Acetyl chloride was distilled. Quinine was purchased from ACROS. Cobalt(II) perchlorate hexahydrate and benzyloxyacetaldehyde were purchased from Aldrich and used as received. Other reagents and solvents were used without further purification. Flash column chromatography was performed on silica gel 60 (EM Science, 230-400 mesh). Infrared (IR) spectra were recorded on a Perkin-Elmer Spectrum GX FT-IR spectrophotometer as a thin film (neat) on $\mathrm{NaCl}$ plates. ${ }^{1} \mathrm{H}$ and ${ }^{13} \mathrm{C}$ NMR spectra were measured on a Varian VXR-400 or Unity INOVA-600 spectrometer. For ${ }^{1} \mathrm{H}$ NMR spectra, chemical shifts $(\delta)$ are reported in parts per million (ppm) relative to TMS $\left(0.00 \mathrm{ppm}\right.$ in $\left.\mathrm{CDCl}_{3}\right)$, or the corresponding residue

$\mathrm{CHCl}_{3}$ solvent peak. ${ }^{13} \mathrm{C}$ NMR spectra were referenced to the center peak of deuterochloroform (77.0 ppm). Mass spectra were recorded on a Hewlett Packard Series 1100 spectrometer by ESI method.

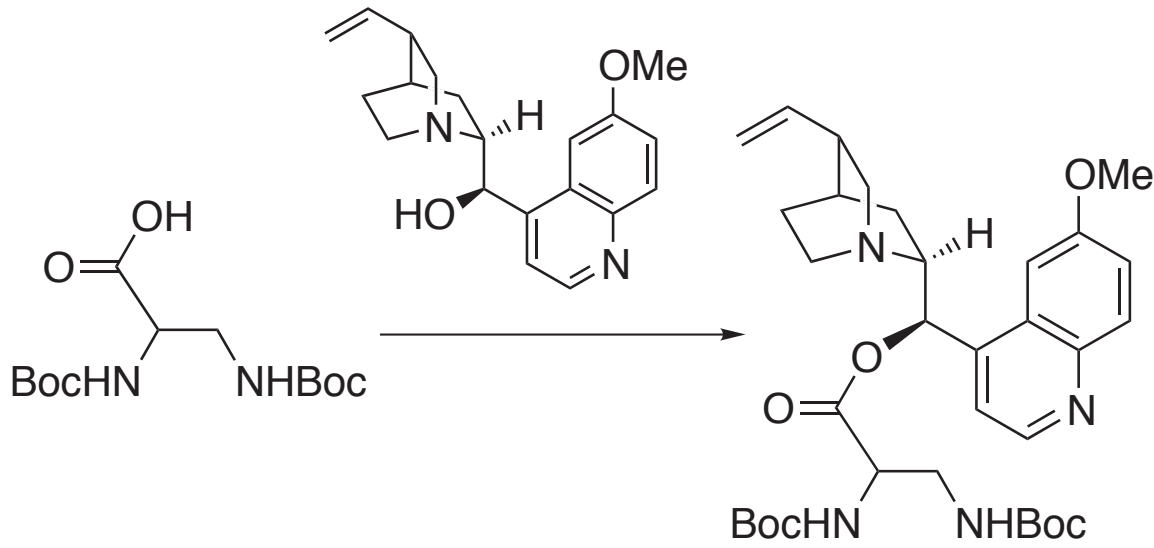

Quinine derived ester. To a solution of racemic $\mathrm{N}^{\alpha}$-Boc- $\mathrm{N}^{\beta}$-Boc-2,3-diaminopropionic acid (10.0 g, 32.9 mmol), quinine (12.0 g, $37.0 \mathrm{mmol})$, and DMAP (1.6 g, $13.1 \mathrm{mmol})$ in methylene chloride $(120 \mathrm{~mL})$ at $\mathrm{rt}$, was added WSC (7.6 g, $39.6 \mathrm{mmol})$. The resulting yellow solution was stirred at $\mathrm{rt}$ for $10 \mathrm{~h}$. The reaction was quenched with saturated sodium bicarbonate solution and the mixture was extracted with methylene chloride. The combined extracts were washed with brine, dried $\left(\mathrm{Na}_{2} \mathrm{SO}_{4}\right)$, filtered, concentrated and separated by flash column chromatography on silica gel ( $1 \%$ to $2 \%$ to $\left.5 \% \mathrm{CH}_{3} \mathrm{OH} / \mathrm{CH}_{2} \mathrm{Cl}_{2}\right)$ to give $17.5 \mathrm{~g}(87 \%)$ of ester as a $1: 1$ mixture of diastereomers.

${ }^{1} \mathrm{H}$ NMR $\left(600 \mathrm{MHz}, \mathrm{CDCl}_{3}\right): \delta 8.72(\mathrm{~d}, J=4.2 \mathrm{~Hz}, 1 \mathrm{H}), 8.70(\mathrm{~d}, J=4.8 \mathrm{~Hz}, 1 \mathrm{H}), 7.99(\mathrm{~d}, J=9.0 \mathrm{~Hz}, 1 \mathrm{H})$, $7.98(\mathrm{~d}, J=9.0 \mathrm{~Hz}, 1 \mathrm{H}), 7.40-7.31(\mathrm{~m}, 6 \mathrm{H}), 6.48(\mathrm{br}, 1 \mathrm{H}), 6.38(\mathrm{br}, 1 \mathrm{H}), 5.83-5.73(\mathrm{~m}, 3 \mathrm{H}), 5.55(\mathrm{br} \mathrm{d}, J=6.0 \mathrm{~Hz}$, 1H), 5.00-4.94 (m, 4H), 4.84 (br s, 1H), 4.71 (br s, 1H), 4.42-4.38 (m, 2H), 3.92 (s, 6H), 3.55-3.32 (m, 6H), 3.07-2.96 $(\mathrm{m}, 4 \mathrm{H}), 2.66-2.54(\mathrm{~m}, 4 \mathrm{H}), 2.24(\mathrm{~m}, 2 \mathrm{H}), 2.00-1.51(\mathrm{~m}, 10 \mathrm{H}), 1.42(\mathrm{~s}, 9 \mathrm{H}), 1.40(\mathrm{~s}, 18 \mathrm{H}), 1.36(\mathrm{~s}, 9 \mathrm{H}) ;{ }^{13} \mathrm{C} \mathrm{NMR}$ 
$\left(100 \mathrm{MHz}, \mathrm{CDCl}_{3}\right) \delta 170.7,170.5,158.2,158.1,157.1,156.9,155.9,155.7,147.7,147.5,144.7,144.69,143.6,143.2$, $141.9,141.8,132.0,131.9,127.1,126.8,122.1,114.8,114.7,101.4,101.2,80.2,80.0,75.6,59.6,59.1,57.0,56.6$, 55.8, 55.78, 55.3, 55.0, 42.9, 42.6, 42.2, 42.1, 39.8, 28.4, 27.8, 27.74, 27.68, 27.6 24.6, 23.6; IR (neat): 3362.2, 1707.6, 1510.6, 1367.0; MS calc. for $\mathrm{C}_{33} \mathrm{H}_{47} \mathrm{~N}_{4} \mathrm{O}_{7}\left(\mathrm{MH}^{+}\right)$: 611.3, found: 611.3. 


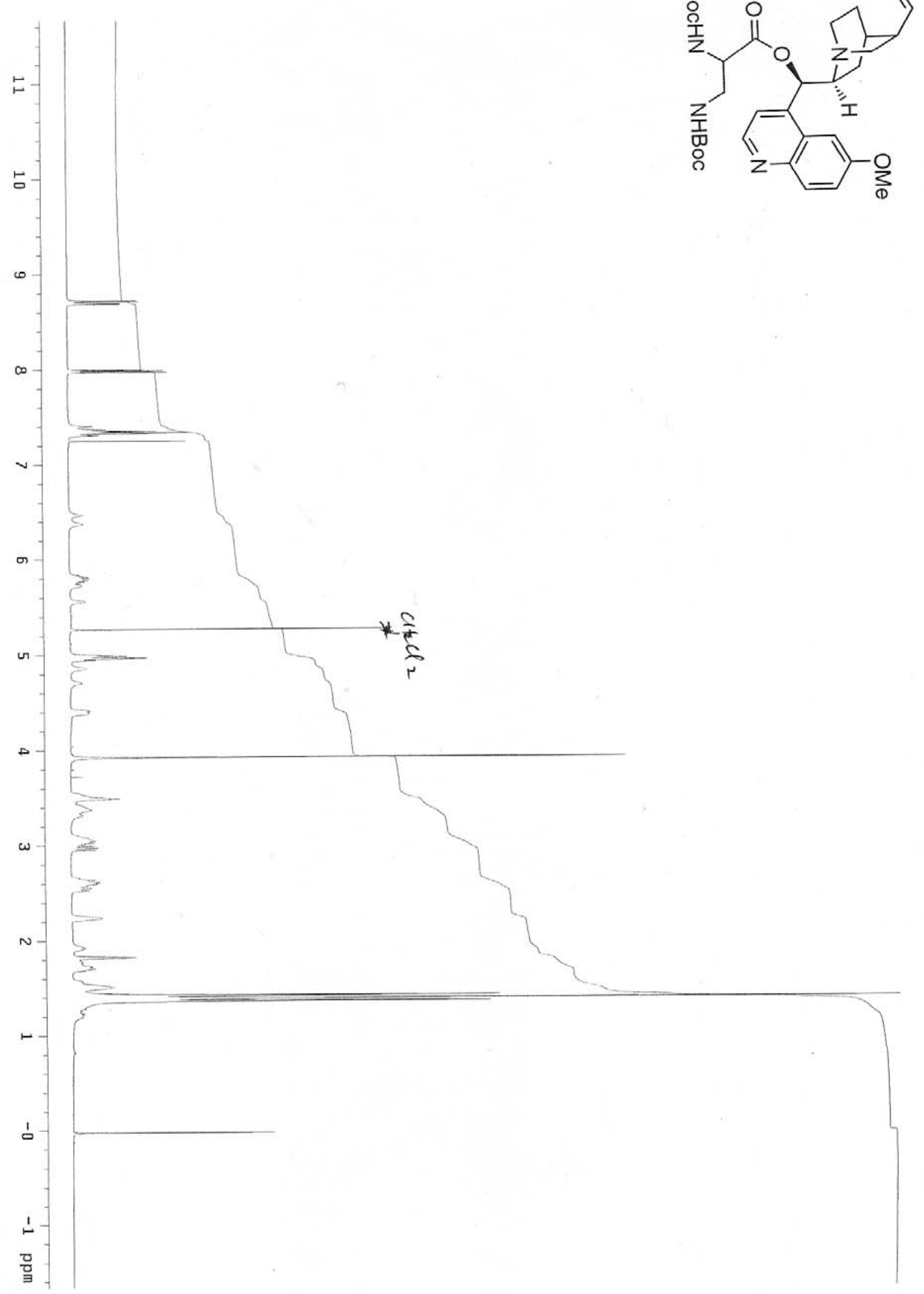




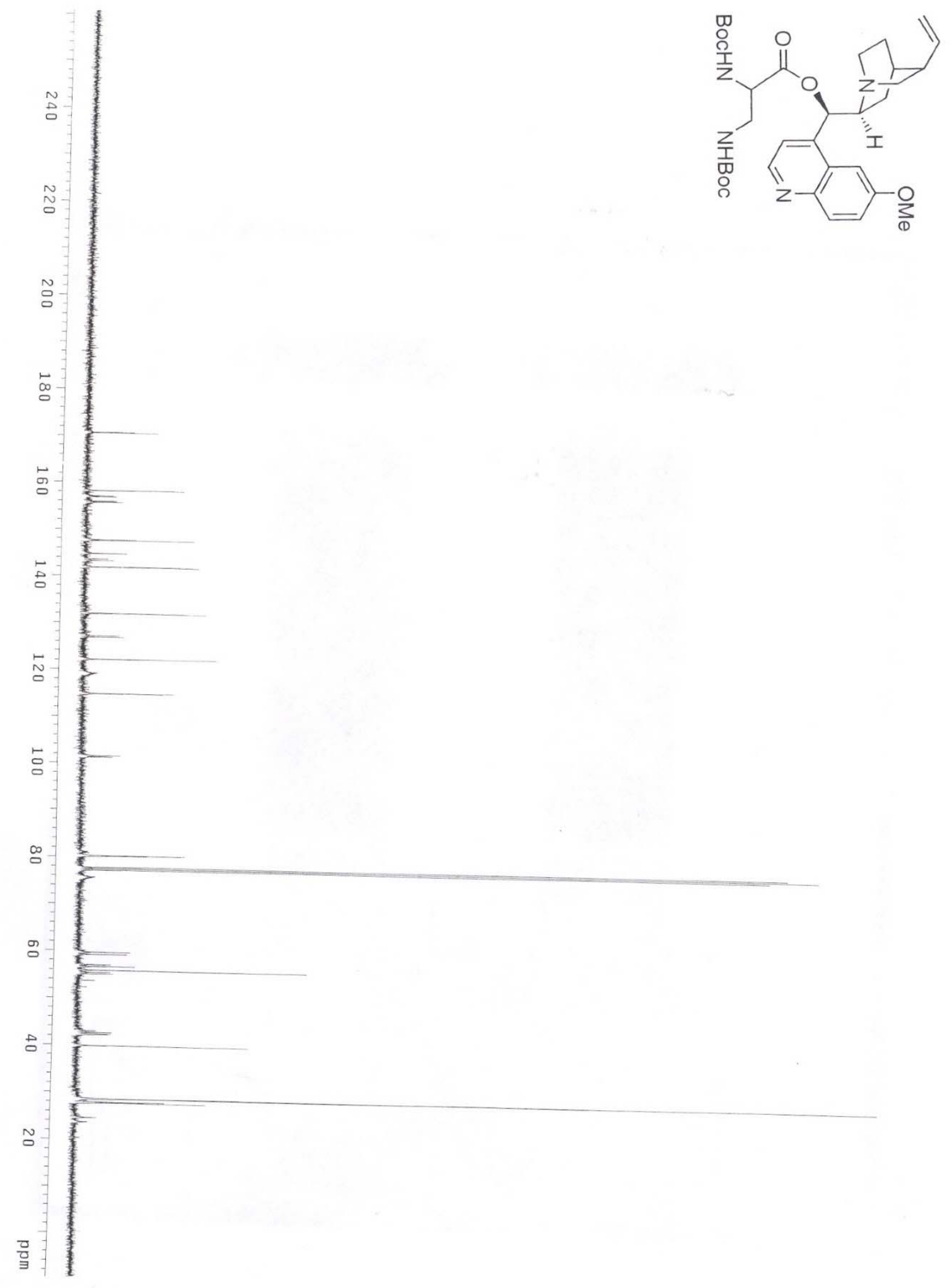




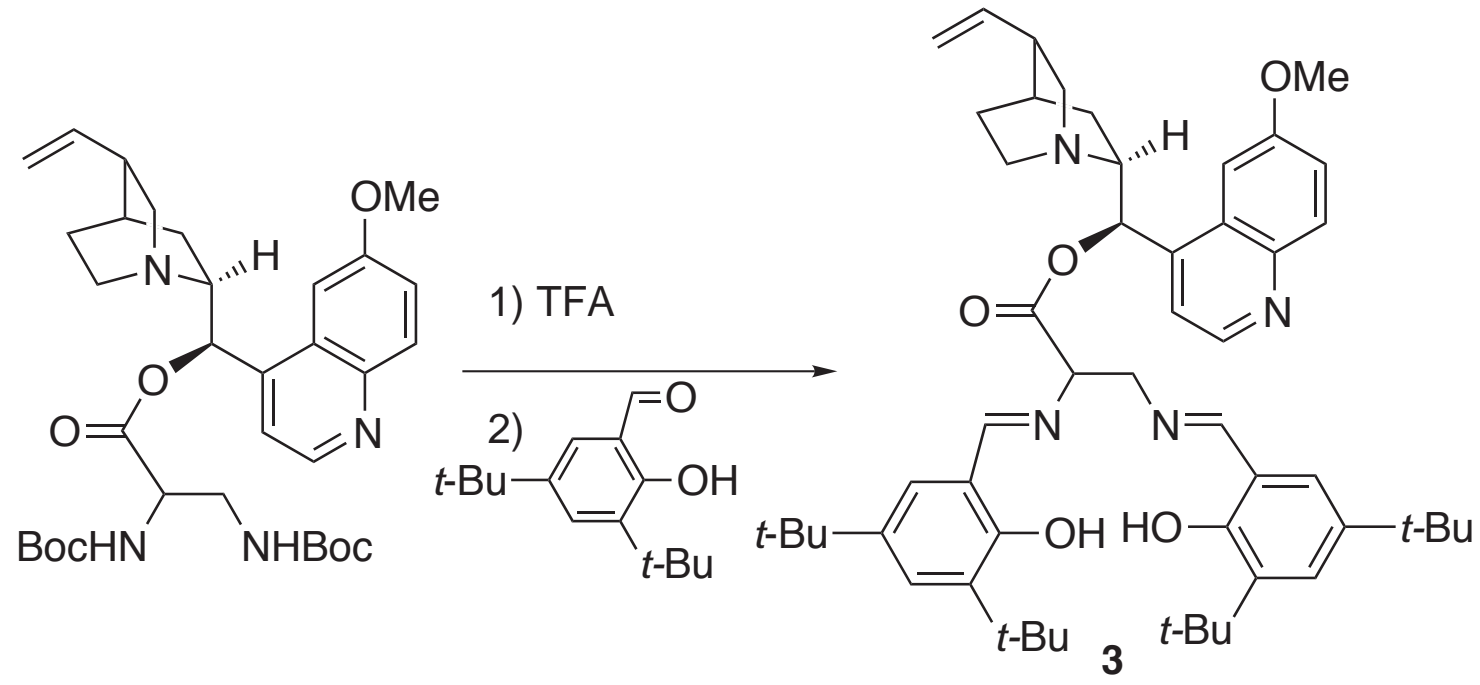

Pentadentate Ligand (3). To a solution of the Boc-protected ester (3.0 g, $4.92 \mathrm{mmol})$ in $\mathrm{CH}_{2} \mathrm{Cl}_{2}(15 \mathrm{~mL})$ at $0{ }^{\circ} \mathrm{C}$, was added TFA $(15 \mathrm{~mL})$. The solution was stirred at $0{ }^{\circ} \mathrm{C}$ for $1 \mathrm{~h}$ and the volatiles were evaporated to give the desired ammonium TFA salt as a sticky oily residue, which was used directly in the following reaction.

To the above TFA salt at $\mathrm{rt}$, was added methylene chloride (150 mL), isopropanol (150 $\mathrm{mL})$, and triethylamine $(9.0 \mathrm{~mL})$. To the resulting solution at rt, was added 3,5-di-t-butyl-2-hydroxybenzaldehyde (2.19 g, 9.36 mmol), and $4 \AA$ molecular sieves. The resulting mixture was stirred for $12 \mathrm{~h}$. The molecular sieves were filtered off and rinsed with methylene chloride. The filtrate was concentrated to give an oil which was dissolved in ethyl acetate, washed with sodium bicarbonate and brine, dried $\left(\mathrm{Na}_{2} \mathrm{SO}_{4}\right)$, filtered, and concentrated to give $3.80 \mathrm{~g}$ of ligand $\mathbf{3}$ (92\% in two steps) as a yellow foam.

${ }^{1} \mathrm{H}$ NMR $\left(600 \mathrm{MHz}, \mathrm{CDCl}_{3}\right) \delta 13.24(\mathrm{~s}, 1 \mathrm{H}), 13.22(\mathrm{~s}, 1 \mathrm{H}), 13.18(\mathrm{~s}, 1 \mathrm{H}), 13.16(\mathrm{~s}, 1 \mathrm{H}), 8.62(\mathrm{~d}, J=4.8 \mathrm{~Hz}$, $1 \mathrm{H}), 8.59(\mathrm{~d}, J=4.8 \mathrm{~Hz}, 1 \mathrm{H}), 8.40(\mathrm{~s}, 1 \mathrm{H}), 8.38(\mathrm{~s}, 1 \mathrm{H}), 8.34(\mathrm{~s}, 1 \mathrm{H}), 8.26(\mathrm{~s}, 1 \mathrm{H}), 7.98(\mathrm{~d}, J=6.0 \mathrm{~Hz}, 1 \mathrm{H}), 7.96(\mathrm{~d}, J$ $=6.6 \mathrm{~Hz}, 1 \mathrm{H}), 7.40-7.30(\mathrm{~m}, 10 \mathrm{H}), 7.04(\mathrm{~d}, J=1.8 \mathrm{~Hz}, 1 \mathrm{H}), 7.00(\mathrm{~d}, J=2.4 \mathrm{~Hz}, 1 \mathrm{H}), 6.99(\mathrm{~d}, J=2.4 \mathrm{~Hz}, 1 \mathrm{H}), 6.96(\mathrm{~d}$, $J=1.8 \mathrm{~Hz}, 1 \mathrm{H}), 6.50(\mathrm{br} \mathrm{s}, 2 \mathrm{H}), 5.77(\mathrm{~m}, 2 \mathrm{H}), 4.97-4.90(\mathrm{~m}, 4 \mathrm{H}), 4.42-4.38(\mathrm{~m}, 2 \mathrm{H}), 4.22(\mathrm{dd}, J=12.6,4.2 \mathrm{~Hz}, 1 \mathrm{H})$, $4.16(\mathrm{dd}, J=12.6,4.2 \mathrm{~Hz}, 1 \mathrm{H}), 3.98-3.89$ (m, 2H), 3.92 (s, 3H), 3.82 (s, 3H), 3.37 (m, 2H), 3.08 (m, $2 \mathrm{H}), 3.02-2.96$ $(\mathrm{m}, 2 \mathrm{H}), 2.64-2.52(\mathrm{~m}, 4 \mathrm{H}), 2.21(\mathrm{~m}, 2 \mathrm{H}), 1.87(\mathrm{~m}, 2 \mathrm{H}), 1.77-1.74(\mathrm{~m}, 2 \mathrm{H}), 1.64(\mathrm{~m}, 4 \mathrm{H}), 1.44-1.40(\mathrm{~m}, 2 \mathrm{H}), 1.43(\mathrm{~s}$ 9H), $1.426(\mathrm{~s}, 9 \mathrm{H}), 1.40(\mathrm{~s}, 18 \mathrm{H}), 1.27(\mathrm{~s}, 9 \mathrm{H}), 1.26(\mathrm{~s}, 9 \mathrm{H}), 1.256(\mathrm{~s}, 18 \mathrm{H}) ;{ }^{13} \mathrm{C} \mathrm{NMR}\left(100 \mathrm{MHz}, \mathrm{CDCl}_{3}\right) \delta 171.4$, $169.8,169.7,169.5,169.4,169.2,158.4,158.3,158.2,158.18,158.17,147.8,147.7,145.0,143.2,143.15,141.9$, $140.73,140.71,140.54,140.52$, 137.11, 137.10, 136.9, 132.2, 132.12, 128.3, 127.8, 127.7, 127.1, 127.0, 126.8, 126.5, 122.2, 117.85, 117.82, 117.74, 117.69, 114.8, 114.7, 101.4, 101.3, 71.74, 74.70, 61.7, 61.6, 60.6, 59.5, 59.4, 56.9, 55.9, 55.7, 42.7, 40.00, 39.97, 35.34, 35.29, 34.4, 31.70, 31.68, 29.7, 27.9, 27.88, 27.6, 24.5, 24.4; IR (neat): 3407.8, 1740.9, 1623.6, 1474.8, 1362.2; MS calc. for $\mathrm{C}_{53} \mathrm{H}_{71} \mathrm{~N}_{4} \mathrm{O}_{5}\left(\mathrm{MH}^{+}\right)$: 843.5, found: 843.8. 


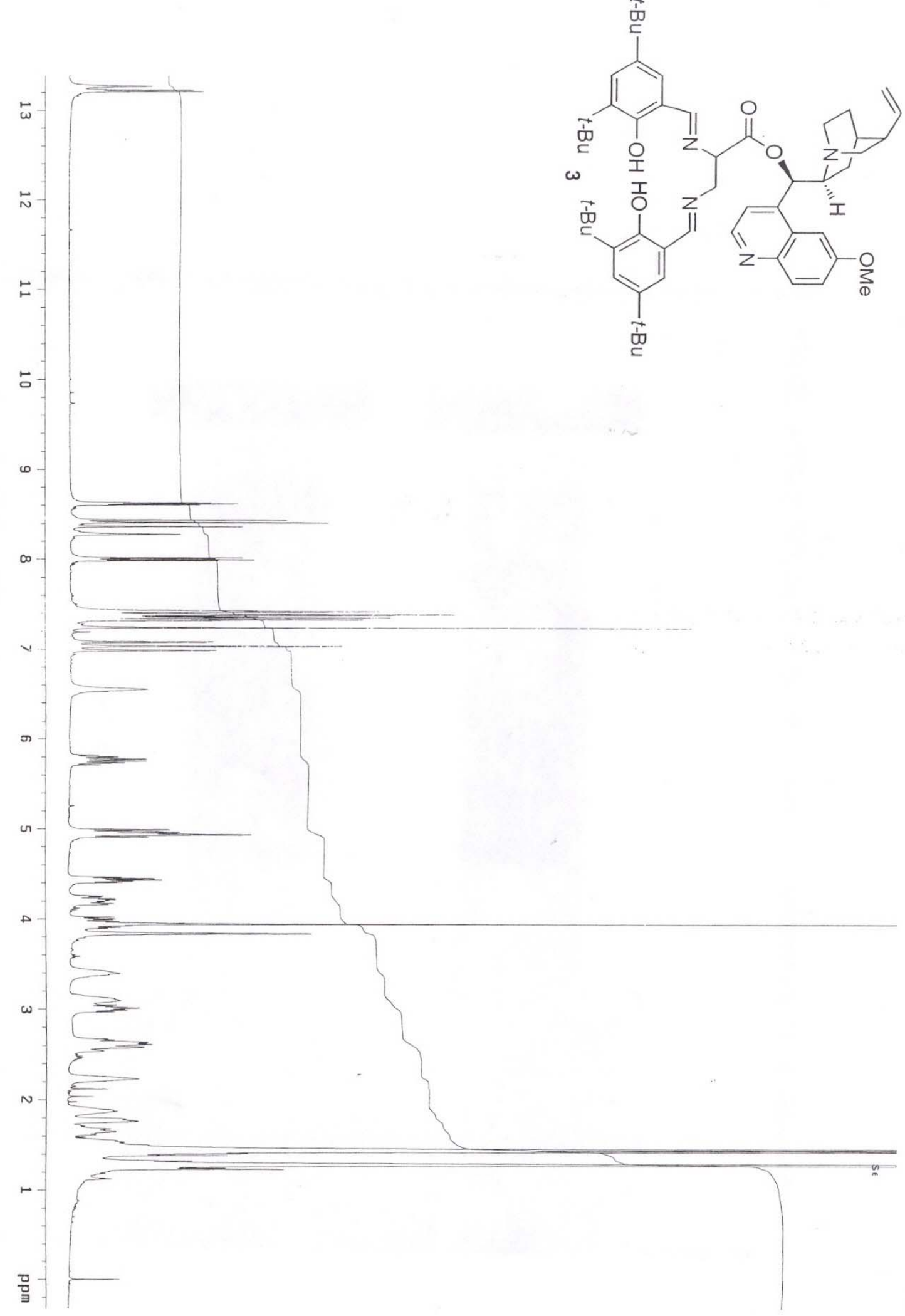<smiles>C=C</smiles>

6 

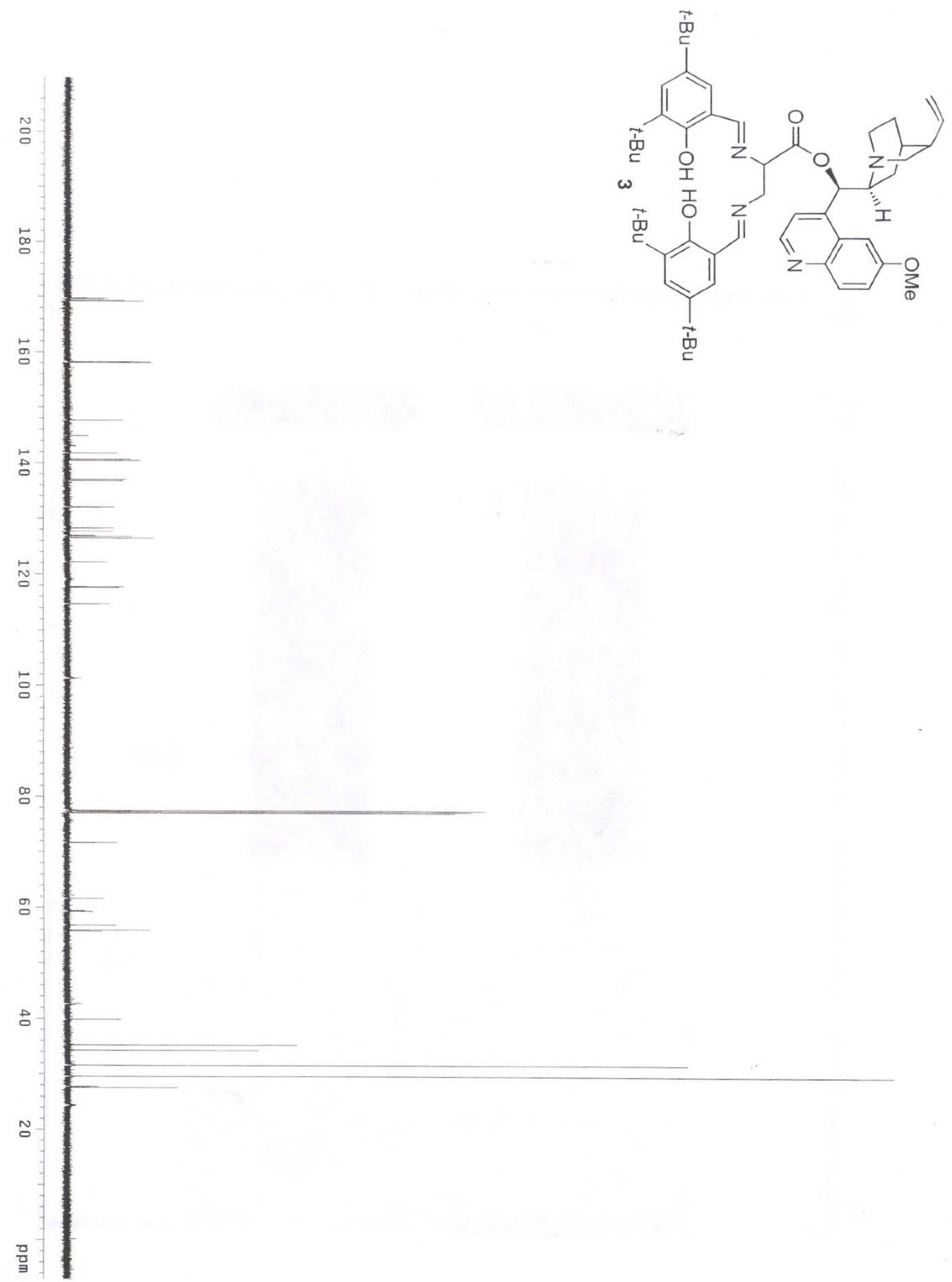
Synthesis of bifunctional catalyst 13 and its catalytic activity To a degassed THF (50 mL) solution of ligand 3 (1.0 g, $1.186 \mathrm{mmol}$ ) at rt, was added cobalt(II) perchlorate hexahydrate (434 mg, $1.186 \mathrm{mmol})$. The resulting mixture was refluxed (oil bath) under Ar for $2 \mathrm{~h}$. The resulting deep red solution was cooled to rt and the volatiles were removed. The residue was dried under high vacuum (ca. 0.1 Torr) for $12 \mathrm{~h}$ to give cobalt (II) complex 13 (1.46 g) as a dark red solid, which was used as the catalyst without further purification. MS calcd. for $\mathrm{C}_{53} \mathrm{H}_{68} \mathrm{~N}_{4} \mathrm{O}_{5} \mathrm{Co}\left(\mathrm{M}^{+}\right)$ 899.4, found, 899.7.

To a solution of catalyst $13(9.0 \mathrm{mg}, 0.010 \mathrm{mmol})$ in methylene chloride $(39 \mathrm{~mL})$ under $\mathrm{Ar}$ at $-78{ }^{\circ} \mathrm{C}$, was added aldehyde $8(0.14 \mathrm{~mL}, 1.0 \mathrm{mmol})$ and diisopropylethylamine $(0.33 \mathrm{~mL}, 2.0 \mathrm{mmol})$. A solution of acetyl chloride $(0.10 \mathrm{~mL}, 1.40 \mathrm{mmol})$ in methylene chloride $(1 \mathrm{~mL})$ was added slowly $(\mathrm{ca} .0 .5 \mathrm{~h})$ to the above solution and the reaction was stirred at $-78{ }^{\circ} \mathrm{C}$ for $1 \mathrm{~h}$. The reaction was quenched with saturated sodium bicarbonate solution and extracted with methylene chloride. The combined extracts were washed with brine, dried $\left(\mathrm{Na}_{2} \mathrm{SO}_{4}\right)$, filtered, concentrated, and separated by flash column chromatography on silica gel (8:1 to 4:1 hexanes/ethyl acetate) to give $159 \mathrm{mg}(83 \%)$ of (4S)-4-Benzyloxymethyl-oxetan-2-one (10). ${ }^{1} \mathrm{H}$ NMR and ${ }^{13} \mathrm{C}$ NMR spectra are in complete agreement with the literature. ${ }^{9}$ The absolute configuration and ee $(>99 \%)$ of $\beta$-lactone $\mathbf{1 0}$ was determined by chiral HPLC analysis using Evans' protocol (column: CHIRACEL OD-H; mobile phase: $20 \%{ }^{\mathrm{i}} \mathrm{PrOH} / \mathrm{hexanes}$; flow rate = $1.0 \mathrm{~mL} / \mathrm{min}$, UV detection set at $210 \mathrm{~nm}$ ), giving only one peak having the retention time at 16.8 min, corresponding to the $S$ enantiomer. ${ }^{9}$

Control ligand 11. To a solution of ligand $3(500 \mathrm{mg}, 0.593 \mathrm{mmol})$ in methanol $(10 \mathrm{~mL})$ at $\mathrm{rt}$, was added diisopropylethylamine $(0.098 \mathrm{~mL}, 0.593 \mathrm{mmol})$. The resulting yellow solution was stirred at $\mathrm{rt}$ for $12 \mathrm{~h}$. The volatiles were removed and the residue was separated by flash column chromatography on silica gel (1\% to $5 \%$ $\left.\mathrm{CH}_{3} \mathrm{OH} / \mathrm{CH}_{2} \mathrm{Cl}_{2}\right)$ to give $192 \mathrm{mg}(59 \%)$ of methyl ester 11 as a yellow oil. ${ }^{1} \mathrm{H} \mathrm{NMR}\left(\mathrm{CDCl}_{3}, 600 \mathrm{MHz}\right) \delta 8.42(\mathrm{~s}, 1 \mathrm{H})$, $8.40(\mathrm{~s}, 1 \mathrm{H}), 7.38(\mathrm{~d}, J=2.4 \mathrm{~Hz}, 1 \mathrm{H}), 7.37(\mathrm{~d}, J=2.4 \mathrm{~Hz}, 1 \mathrm{H}), 7.08(\mathrm{~d}, J=2.4 \mathrm{~Hz}, 1 \mathrm{H}), 7.06(\mathrm{~d}, J=2.4 \mathrm{~Hz}, 1 \mathrm{H}), 4.36$ $(\mathrm{dd}, J=7.8,4.2 \mathrm{~Hz}, 1 \mathrm{H}), 4.28(\mathrm{dd}, J=12.6,4.2 \mathrm{~Hz}, 1 \mathrm{H}), 3.95(\mathrm{dd}, J=12.6,7.8 \mathrm{~Hz}, 1 \mathrm{H}), 3.80(\mathrm{~s}, 3 \mathrm{H}), 1.43(\mathrm{~s}, 9 \mathrm{H})$, $1.41(\mathrm{~s}, 9 \mathrm{H}), 1.27(\mathrm{~s}, 18 \mathrm{H}) ;{ }^{13} \mathrm{C} \mathrm{NMR}\left(\mathrm{CDCl}_{3}, 100 \mathrm{MHz}\right) \delta 170.3,169.5,168.7,158.0,157.9,140.3,140.1,136.7$, 136.6, 127.8, 127.3, 126.6, 126.2, 117.6, 117.5, 71.2, 61.3, 52.6, 35.0, 34.99, 34.1, 31.4, 31.38, 29.4, 29.35; IR (neat): 1746.1, 1628.6; MS calc. for $\mathrm{C}_{34} \mathrm{H}_{51} \mathrm{~N}_{2} \mathrm{O}_{4}\left(\mathrm{MH}^{+}\right)$: 551.8, found: 551.5. 


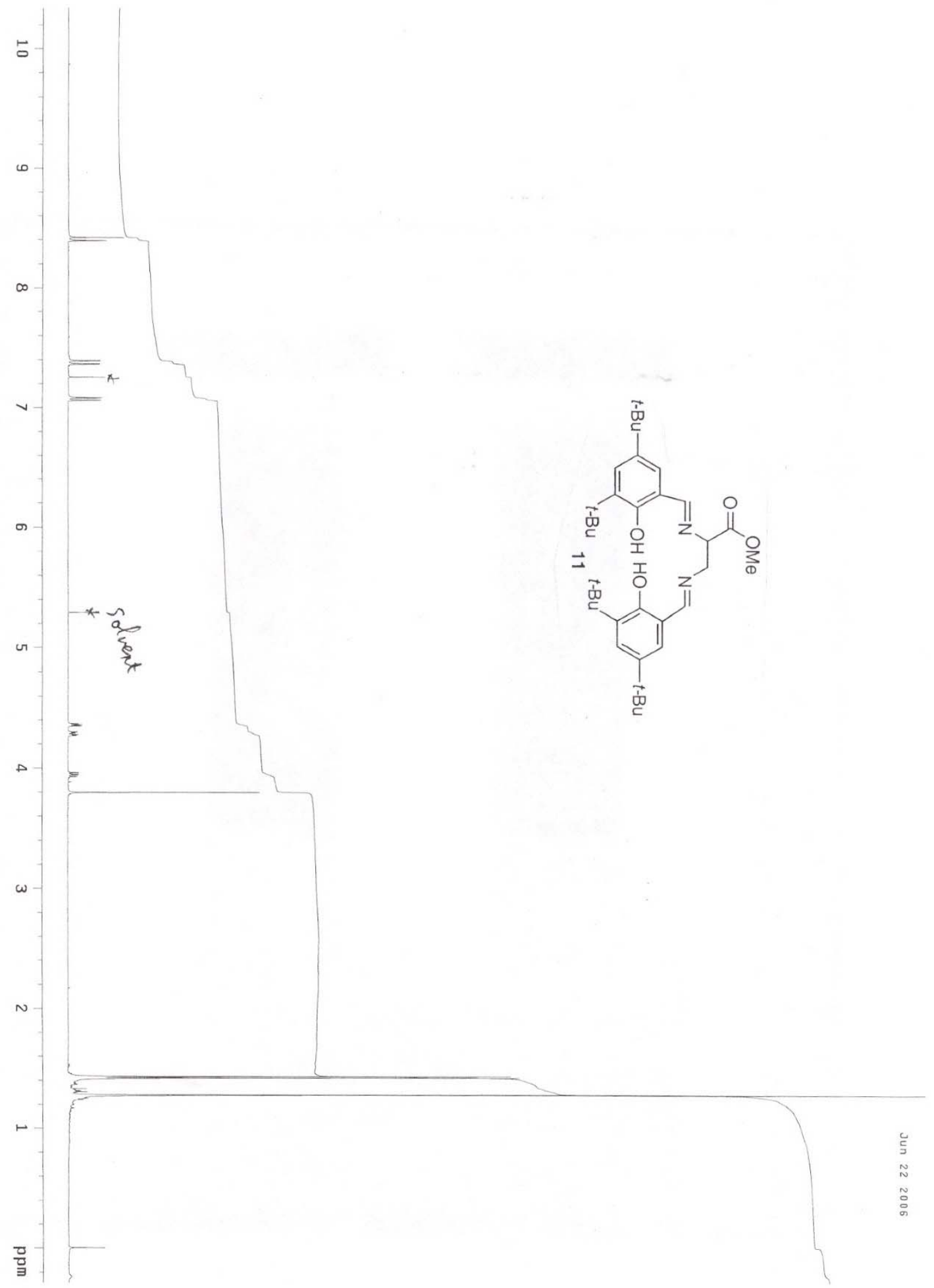




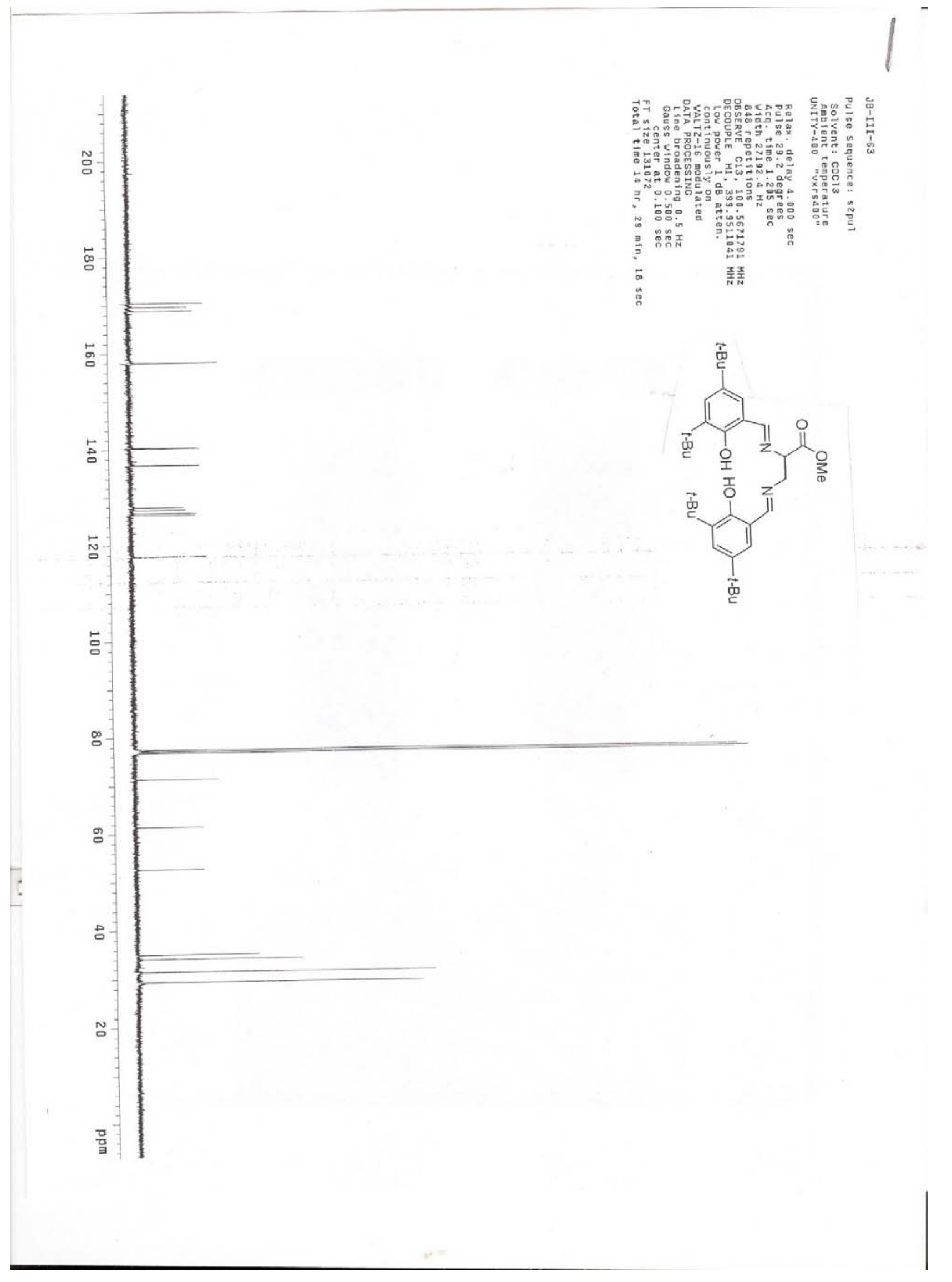




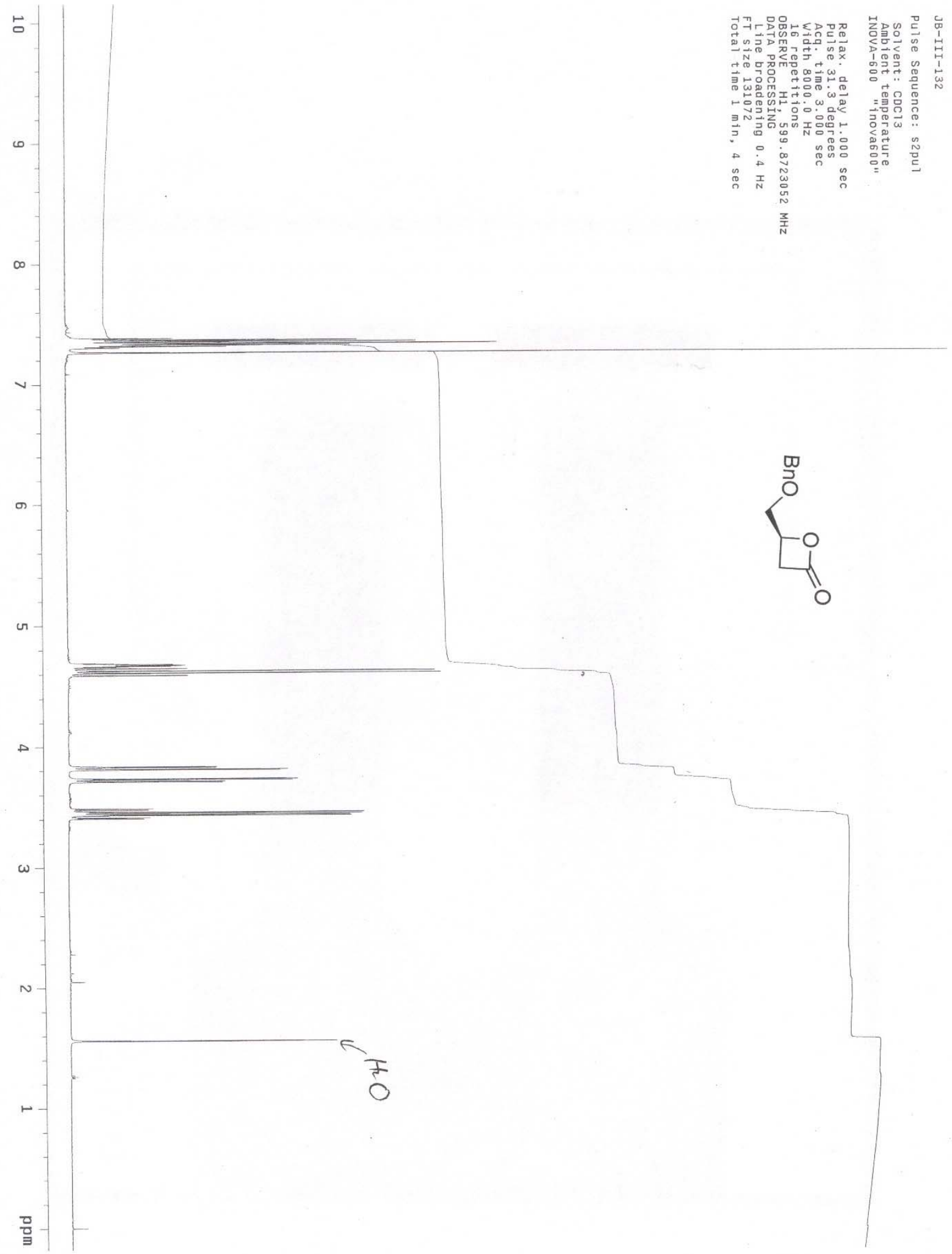




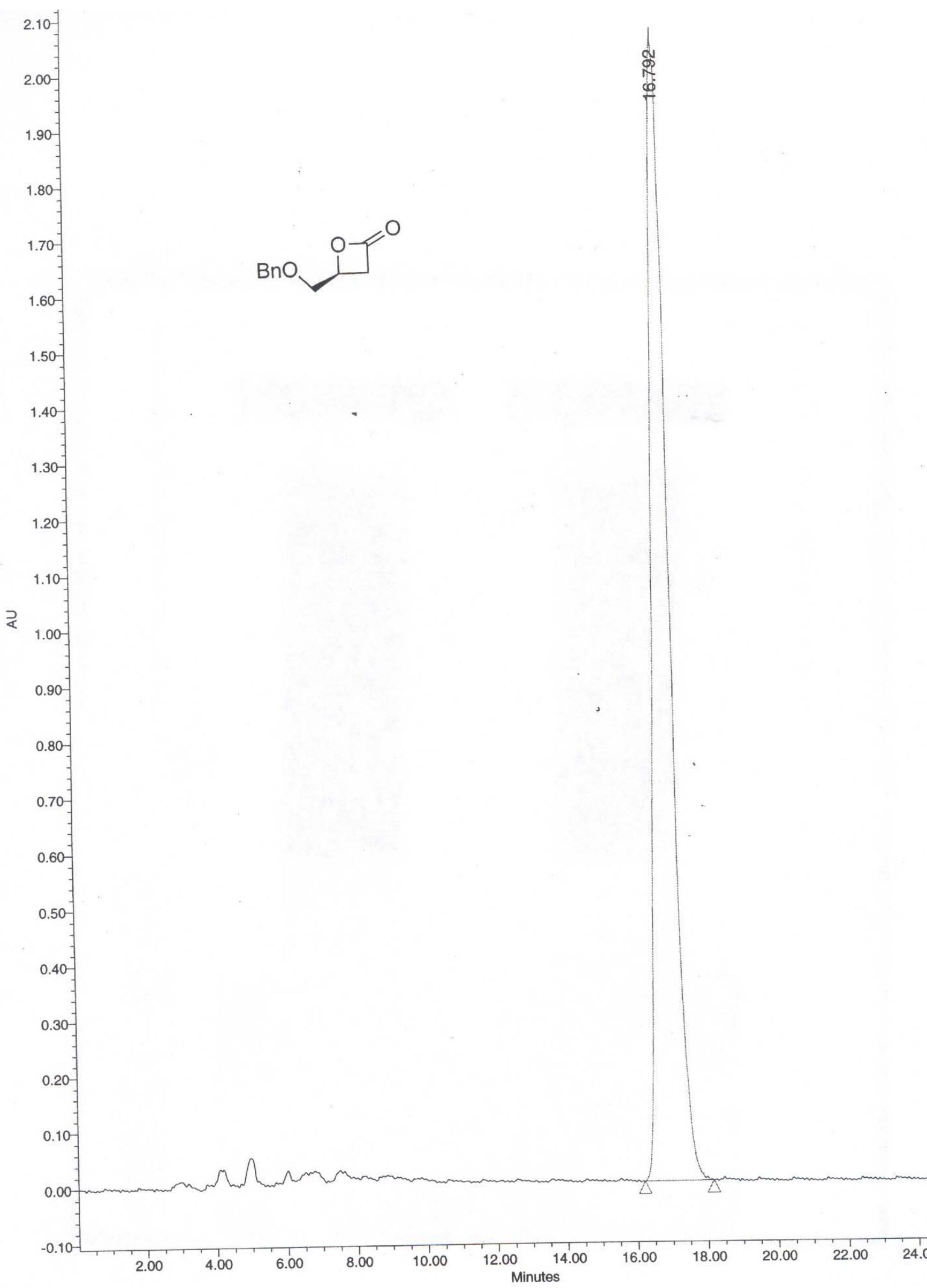

